


\title{
Lengua(je)s, branding e identidad en la Gran Cuba: Dreaming in Cuban de Cristina García
}

\section{Language(s), branding and identity in the Greater Cuba: Cristina García’s Dreaming in Cuban}

Francisca Aguiló Mora Universidad de Columbia, Estados Unidos

\begin{abstract}
Las metáforas conceptuales de puentes, fronteras y otros espacios intermedios -las cuales forman lo que podríamos denominar 'la ontología del guion'- prevalecen en las lecturas críticas de la producción artística de las hijas de la diáspora caribeña hispanófona en los EEUU. No obstante, en este artículo sostengo que las formas lingüísticas y los patrones discursivos que tienen lugar con frecuencia en estos textos no sugieren una carga de identidad lingüística ni el estado de hallarse entre lenguas y naciones, sino que crean una estética de la multiplicidad. A pesar de estar escritas mayoritariamente en inglés, estas obras se agrupan con una tradición literaria del Caribe hispanófono que cuestiona nociones estructuralistas de lengua e identidad, y perspectivas modernas de nación(alidad). En este artículo, expongo la necesidad de reconsiderar las conceptualizaciones de lengua y género en las construcciones de identidades locales, globales y posnacionales en la Gran Cuba. Analizo cómo escritoras como Cristina García (y Achy Obejas, entre otras) reinterpretan la lengua a través de cruces lingüísticos, formando así una comunidad grancaribeña de práctica literaria que cuestiona los límites discursivos de las definiciones tradicionales de 'lo americano', 'lo cu-
\end{abstract}


bano', 'lo caribeńo' y 'lo cubano-americano'. Por medio de su branding lingüístico y sus componentes temáticos, estas novelas desestabilizan los imaginarios nacionales y archivos culturales predominantes tanto en la isla caribeńa como en los EEUU, a la vez que problematizan el rol históricamente silenciado de las mujeres (escritoras), con un claro propósito de adquisición de poder.

Palabras clave: lengua, género, identidad, multiplicidad, guion.

Resumen: The pervasive conceptual metaphors of bridges, borders and other in-between spaces -forming what we may call 'the ontology of the hyphen'- have traditionally prevailed in the readings of the artistic production by the daughters of the Hispanophone Caribbean diaspora in the US. However, I maintain that the linguistic forms and discursive patterns evident in some of these texts are not suggestive of burden of linguistic identity or the state of being between languages and nations, but rather perform an aesthetics of multiplicity within their own right. Although written mostly in English, these works group together with a Hispanophone Caribbean literary tradition to fundamentally question traditional structuralist notions of language and identity, and Modern views of nationhood. In this article, I argue for a much-needed reconsideration of language and gender in the constructions of local, postnational, and global identities in the Gran Cuba. I explore the ways in which writers such as Cristina García (and Achy Obejas, among others) reinterpret language through practices of language crossing, thus creating a grancaribeña community of literary practice that contests the linguistic boundaries of US, US-Latina, US-Caribbean or Cuban discourses as traditionally defined. Through both their language branding and thematic components, these novels destabilize national imaginaries and cultural archives of both the US and the Caribbean island, problematizing the traditional silenced role of women, and women writers specifically, with a clear purpose of power acquisition.

Keywords: Language, Gender, Identity, Multiplicity, Hyphen.

Recibido: 15 de febrero de 2018

Aceptado: 01 de abril de 2018 
... our sense of what it means to be a woman, of how women look, talk, think, feel, comes from the books we read, the films we watch, and the invisible ether of everyday assumptions and cultural beliefs in which we are suspended. Rather than subjects producing texts, in other words, texts produce subjects

FELSKI (2000: 181).

$Y^{2}$

a en las últimas décadas del siglo XX, una corriente de escritoras -hijas de la diáspora caribeńa en los EEUU- publican obras literarias que tratan cuestiones de lengua, género, identidad, sexualidad, etnicidad, desplazamiento, memoria, exilio, nación y comunidad. Las ubicuas metáforas conceptuales de puentes, fronteras $\mathrm{u}$ otros espacios intermedios han prevalecido en las lecturas críticas de esta producción artística, creando así lo que podríamos denominar 'la ontología del guion'. No obstante, estos textos van más allá del puente y de "la rayita" (Cf. Pérez-Firmat, 1995a; 2000) pues, tal como argumenta Lillian Manzor al definir el teatro usanocubano, no se basan en un concepto estático y homogéneo de una identidad nacional o cultural opuesta o separada de otra cultura (VIII), como pareciera sugerir la noción binaria del guion de Gustavo Pérez-Firmat. ${ }^{1}$ Por el contrario, desestabilizan las cul-

${ }^{1}$ Pérez Firmat expone abiertamente este sentido de dualidad como un vaivén traumático (1995b: 201) que ocurre no sólo a nivel identitario sino también lingüístico (2000: lección 17), de ahí la figura del signo ortográfico. Si bien el escritor da un paso muy importante a la hora de cuestionar el discurso nacional canónico centrado en la isla de 'lo cubano' y redefinir las connotaciones negativas de la teoría de asimilación cultural que proclamaran la pérdida de la cultura de origen en los inmigrantes de segunda y tercera generación en los EEUU (Duany, 2000: 30-31), tanto la metáfora del guion como sus ideas de aposicionalidad y biculturalidad no ayudan a desarticular narrativas dominantes en cuestiones de etnicidad, género y clase social, y siguen ofreciendo una 
turas nacionales predeterminadas a cada lado del guion "a través de la inserción de la diferencia, del 'otro' dentro del cuerpo nacional político, [un cuerpo que se compone del sujeto] cubano isleño y el cubano diaspórico[,] el hegemónico usano [y] el emergente latino en Estados Unidos" (Manzor, 2005: VIII). Aunque estén escritas mayoritariamente en inglés, estas obras forman parte de una tradición literaria del Gran Caribe hispanófono que cuestiona tanto nociones estructuralistas de lengua e identidad como visiones modernas del concepto de nación(alidad).

El Gran Caribe literario y cultural que imagino ofrece la posibilidad de descolonizar imaginarios nacionales que se atribuyen a las identidades caribeñas y, asimismo, a las denominadas identidades con guion de los EEUU. Por un lado, estas escritoras deconstruyen con/en sus textos traumas diaspóricos y memorias colectivas impuestas por sus predecesores. Por otro, valoran y recuperan la herencia caribeña que imaginan, como estrategia de desestabilización de macro-narrativas culturales. Haciendo eco de lo que señala la artista y escritora Coco Fusco en su libro English is Broken Here acerca de la relación entre cubanos en la isla y en la diáspora, sostengo que autoras grancaribeñas en los EEUU y en las islas (como Cristina García, Achy Obejas, Julia Álvarez, Jennine Capó Crucet, Mayra Santos Febres, Aurora Arias, Josefina Báez o Caridad Svich, entre otras), comparten "a healthy skepticism toward the nationalist rhetoric of [their] parents' generation and a growing curiosity

conceptualización binaria del sujeto cubano-americano. Por otro lado, el uso del puente que propone Ruth Behar en Bridges to Cuba concibe un espacio más abierto de reconciliación y renovación entre Cuba y la diáspora estadounidense, lejos de las metáforas naturales -y naturalizadas- de raíces, trasplantes y la Madre Tierra (Cf. Duany, 2000), así como del pensamiento polarizador de la Guerra Fría (Behar, 1995: 5). Pero perpetúa un sistema dual divisorio que sigue sin cuestionar categorías culturales y literarias basadas estrictamente en preceptos nacionalistas (vid. Manzor, 1995: 253) y, consecuentemente, masculinistas. 
about one another" (1995: 5). Estas escritoras visitan o re-visitan las islas -o los EEUU- en busca de una red de creación artística en el Gran Caribe que, como ellas, no se identifica con los modelos monolíticos descriptivos (Fusco, 1995: 13) y añadiría, prescriptivos, de imaginarios culturales y nacionales. Sus obras se distancian de la epistemología heredada que se apoya en el principio de la dualidad (tanto en los EEUU como en las islas): "real identity versus fake identity, original versus copy, upper class versus working class, good Spanish versus bad Spanish" (1995: 15). Precisamente por estas razones, en mi análisis agrupo a aquellas autoras que escriben desde los EEUU y desde el Caribe bajo la conceptualización artística de Gran Caribe, un espacio cultural heterogéneo y translocal. ${ }^{2}$ En dicha conceptualización, el Caribe conecta con islas vecinas no necesariamente hispanófonas y sus diásporas. ${ }^{3}$ Específicamente, me refiero a la Gran Cuba como un espacio geopolítico, cultural y sociolingüístico en el cual desdibujo la dicotomía los de aquí / los de allá entre EEUU y la isla. ${ }^{4}$

${ }^{2}$ Adopto la definición de translocalidad de Mayra Santos Febres como una herramienta que define las prácticas sociales y discursivas de escritores que se hallan bajo condiciones de migración circular. Esta idea difiere de los conceptos de hibridismo y conciencia oposicional en tanto que se centra en cuestiones de emplazamiento y desplazamiento dentro de contextos multinacionales. La noción de translocalidad se aplica a aquellos textos que responden a una multiplicidad de áreas discursivas que cuestionan opresiones múltiples en relación a identidades raciales, nacionales, sexuales, de género y de clase social (1993: 176).

${ }^{3}$ Aunque reconozco las dimensiones criollas, francófonas y anglófonas del Caribe, los textos que aquí examino se relacionan específicamente con el Caribe hispanófono. Una definición precisa del Gran Caribe incluye las múltiples lenguas y acentos que lo componen, no siempre correspondientes con límites geopolíticos delimitados.

${ }^{4}$ Otros académicos adoptan una postura similar a la que aquí presento. Por ejemplo, Rodríguez-Mourelo en su análisis del desplazamiento cultural en textos literarios 'cubano-americanos' señala que "the responses to representations of displacement I am seeking cannot be seen as a contraposition of inside/outside; 
A diferencia de la idea de vivir entre idiomas o de bailar entre culturas (Cf. Luis, 1997), las escritoras grancaribeñas a las que he hecho referencia habitan varias lenguas y múltiples lenguajes. Indudablemente, mientras que ciertos textos de los denominados 'US Latino' -o más específicamente, de los 'cubano-americanos'permanecen en este espacio intermedio que propone la metáfora del guion, y mientras que ciertas obras de autoras hispano caribeñas encajan en macro-narrativas fijas de género y nación, la literatura que menciono y analizo cruza lengua(je)s y acentos, y habita espacios múltiples y fluidos. Así, la primera sección del presente artículo propone que, concretamente a través de la práctica discursiva del cruce lingüístico, esta literatura manifiesta movimiento y una estética de la multiplicidad. Esta práctica es a la vez la conceptualización que adopto como herramienta de análisis ya que expongo que el estudio harto común de la variación local estática en la sociolingüística de distribución, junto con los conceptos de hablante nativo, lengua materna, home(land) y doubled selves, nos lleva inevitablemente a equívoco. Además, muestro que el cruce lingüístico presente en estas obras se convierte en un branding ideológico que otorga visibilidad a la históricamente silenciada mujer escritora en el mercado editorial. A través de un análisis crítico discursivo, entendiendo la naturaleza relacional y transdiciplinaria del discurso escrito, considero cuestiones de producción y consumo en relación a las prácticas lingüísticas mencionadas y la forma en que estas desestabilizan cánones y tradiciones literarias. Examino, asimismo, cómo dicha virtuosidad discursiva se presenta

nor merely as a 'bridge' or 'hyphen' as these images imply a two-sided concept and can be applied only to the Cuban American experience (2006: 27). Asimismo, Jorge Duany requiere discursos más fluidos que trasciendan el territorio insular, las definiciones jurídicas de ciudadanía y nacionalidad, las posturas tradicionales de ideología política en el contexto cubano-americano e incluso la oposición estándar entre el español y el inglés (2000: 36). 
y organiza en relación al contexto socio-ideológico grancaribeño de los textos, sus autoras y su imaginada reformulación de grandes narrativas monolingües y monoculturales, así como 'masculinistas', en el Gran Caribe. Para ello, me detengo brevemente en la Gran Cuba con las novelas debut de Cristina García, Dreaming in Cuban (1993), y Achy Obejas, Memory Mambo (1996). Finalmente, teniendo en cuenta que el contenido de los textos acompaña a su forma lingüística en lo que se refiere al afán de sus protagonistas femeninas por cuestionar significados se identidades fijas y representaciones modernas de 'lo americano', 'lo cubano' y 'lo cubano-americano', en la segunda sección llevo a cabo un análisis crítico-literario de la primera novela de García, relacionando cuestiones de lengua(je) e identidad con Pilar, la protagonista. A nivel formal, esta novela reinterpreta la lengua a través de la noción del cruce lingüístico, y a nivel temático busca lengua(je)s alternativos, de modo que funciona como una proyección que altera la realidad $\mathrm{o}$, al menos, la tendencia discursiva predominante en cuestiones de lengua, nación(alidad) y género.

\section{Cruces lingüísticos: un branding ideológico más allá del guion}

La crítica implícita en los textos de estudio de un guion que divide imaginarios nacionales establecidos va de la mano de otros conceptos no menos engańosos y poco transparentes: los de hablante nativo y lengua materna y, en consecuencia, la indisoluble asociación entre estos términos con el lugar de nacimiento - home(land) - y la nación(alidad).

Desde el campo de la lingüística aplicada, Alan Davies reflexiona acerca de la dificultad que conlleva definir qué es un hablante nativo. Davies admite que la distinción entre hablante nativo y no nativo refleja el principio chomskiano del sujeto nativo idea- 
lizado -el cual se concibe monolingüe y sirve como modelo de una competencia gramatical universal basada en estructuras lingüísticas mentales innatas que a la vez ignora factores sociales y realidades contextuales heterogéneas-. Obviamente, este hablante nativo 'ideal' que produce una lengua igualmente 'ideal' habita una comunidad de habla estática y homogénea. El estructuralista Leonard Bloomfield usa el término 'lengua nativa' para hacer mención a la L1 (primera lengua), i.e. "sithat one learned at one's mother's knee" (435), definición que resulta imprecisa en las autoras grancaribeñas que escriben desde los EEUU. En ellas, el uso de su 'primera lengua' o 'materna', el español, se detiene a la edad de la escolarización en los EEUU (Cf. Lynch, 2012: 82; Piller, 2001: 5). ¿Cuál es entonces la L1 en estos casos? ¿Es igual o diferente a la lengua materna? Teniendo en cuenta las habilidades lingüísticas de las literatas, ¿̇no podríamos considerar que es el inglés su primera lengua, a pesar de que el español fuera la materna? (Cf. MartínezSan Miguel, 2003: 372). En el campo de la sociolingüística y la lingüística aplicada, habría un claro acuerdo a la hora de considerar a estas autoras hablantes de español como lengua de herencia, ya que algunas nacieron y todas se instruyeron formalmente en los EEUU y, aunque de ascendencia hispanohablante, el uso del español en el ámbito familiar es limitado en la mayoría de los casos (Cf. Lynch, 2003: 30).

Por todo ello, escriben principalmente en inglés. Este es el idioma con el que se formaron intelectualmente, y para la mayoría de ellas existe una clara conexión entre la adquisición del inglés y su desarrollo como escritoras (del Rio, 2008:19). En la obra Conversations with Cuban-American Writers -compilación que incluye entrevistas con Cristina García y Achy Obejas- Eduardo del Rio propone que, para algunas de ellas, el español deviene un mero recuerdo o un fenómeno lingüístico que han experimentado esporádicamente a través de lazos familiares. Añade que, para todas 
ellas, "their linguistic consciousness includes a sense of both languages. Because of this duality, the body of Cuban-American writers works is written primarily in English, as they seek to express this conflict in the language that is the embodiment of it" (2008: 4). La idea de que, para autoras como Obejas y García, el bilingüismo constituye un conflicto, el cual personifican con el uso del inglés en sus obras, perpetúa la noción de lucha entre los de aquí y los de allá y entre sus dos idiomas y los imaginarios nacionales que cada una de estas lenguas encarnan desde la perspectiva estructuralista dominante del siglo XX; i.e. la idea de un Estado-nación lingüísticamente homogéneo (Cf. Heller, 2007). Por el contrario, la narrativa grancaribeña se aleja de representaciones de "doubled selves” (Pavlenko, 2006) -la idea de poseer dos identidades incompatibles- y de la imagen del sujeto bilingüe como dos monolingües en un solo cuerpo (vid. también Grosjean, 1982). El trauma de la dualidad -identificada como una esquizofrenia lingüística en la tradición literaria del guion y acompañada por términos tales como bifurcación, bigamia, ruptura, separación, hueco o espacio intermedio, entre otros- apareció en la primera mitad del siglo XX y todavía tiene vigencia en algunos casos (Pavlenko, 2006: 5). ${ }^{5}$ No obstante, ha disminuido en las obras literarias de la modernidad tardía a favor de cuestionar y desarticular nociones esencialistas del 'yo', y sugerir que "anxieties over an inner split may stem from the lack of social acceptance of bilingualism and may disappear once

${ }^{5}$ Por ejemplo, la voz narrativa en primera persona de Silent Dancing, de Judith Ortiz Cofer, describe su identidad intermedia como una esquizofrenia cultural (1990: 34): "Cold/hot, English/Spanish; that was our life" (1990: 129), explica. De igual forma, Pérez-Firmat expresa esta idea de poseer dos identidades incompatibles: "A veces me ha parecido que entre el $Y o$ y el $I$ inglés se abre un abismo imposible de salvar, porque al nombrarnos en una lengua u otra proyectamos o engendramos identidades diversas, tal vez irreconciliables" (2000: lección 17). 
bi- and multilingualism are accepted as the norm, rather than an exception" (Pavlenko, 2006: 28). ${ }^{6}$

Los textos a los que aquí me refiero se afanan por naturalizar el multilingüismo como forma de expresión literaria a pesar de que, por estar escritos en su mayor parte en inglés, la crítica literaria los ha acusado de falta de autenticidad y de representación inadecuada de la cultura (cubana) y lengua (española) de origen de las autoras. Lori Ween explica que una de las respuestas críticas los ha definido como "translational backformations" (2013: 128), fenómeno que se refiere al resultado lingüístico que se obtiene al traducir un texto a su supuesto idioma 'original'. Esta postura asume que escritoras como García u Obejas piensan en español mientras escriben sus obras en inglés a través de un proceso de traslación. Por consiguiente, una futura traducción de sus novelas al español resulta en una suerte de traducción regresiva por medio de la cual el significado 'original' del texto es re-creado y re-codificado. Este enfoque es claramente reduccionista y desdeńa algunos de los aspectos socio-culturales e ideológicos más importantes que se hallan tras las prácticas lingüísticas de esta producción artística, y que aquí exploro. Tal como declara Carolina Hospital en su prólogo a Los atrevidos:

When we categorize writers, especially in today's fluid socio-political reality, (with so many artists in exile) we must resist the temptation to simplify. Writers cannot be defined simply by their choice of

\footnotetext{
${ }^{6}$ Con esta cita, Aneta Pavlenko se refiere a nivel general al trabajo de la generación de escritores que creció en los sesenta/setenta, "witnessing the revival of ethnic consciousness and experiencing the influence of postmodern thought" (2006: 28). Yo opto por no agrupar a las escritoras grancaribeńas según patrones generacionales, sino que lo hago en términos de estrategias lingüísticas, estéticas e ideológicas compartidas. De hecho, no todos los escritores que crecieron en las décadas de los sesenta y setenta han abandonado 'el trauma de la dualidad' o 'la esquizofrenia lingüística'.
} 
language, or their place of birth or residence. A detailed study of the cultural, social and linguistic legacies prevalent in the texts is crucial. Most writers today, especially in the United States, cannot be pigeonholed into a single national identity [or in a national identity at all]" (1988: 17; El énfasis es mío).

La propia Obejas advierte que en Cuba se la ha querido incluir en las letras cubanas pero que el hecho de que escriba en inglés ha supuesto un obstáculo. A ello responde la escritora que (el uso de) la lengua no es un fenómeno estático y nos recuerda que "It's not that people are bilingual, or culture dominant, or whatever, but, more specifically, that they're educated in the United States" (del Rio, 2008: 90). Por su parte, García revela que, por una cuestión de practicidad, el inglés es la lengua 'original' en la cual conceptualiza su creación. Admite que incluye en los diálogos de sus novelas "pockets of Spanish" (Lynch, 2011) pero que esto no significa que piense o que quisiera escribir en este idioma. Ella escribe en un inglés "that is informed by the kind of underground river of Spanish and that tries to accommodate its particular rhythms and surges and syncopations ... so that it captures some Spanish" (Lynch, 2011). Para estas autoras grancaribeñas, el cruce de lengua(je)s deviene una forma imaginada de transformar el mundo que las rodea.

La noción de cruce lingüístico proviene del concepto de language crossing en el trabajo de Ben Rampton. ${ }^{7}$ El sociolingüista define esta idea como el uso de lenguas o variedades lingüísticas que popularmente se asocian a grupos sociales o étnicos a los que el hablante no se supone que pertenezca. De este modo, este fe-

${ }^{7}$ En su estudio sobre las prácticas lingüísticas y las variedades discursivas de jóvenes adolescentes en Gran Bretaña -a través de las cuales estos participantes desestabilizan las identidades étnicas tal como se imaginan en el discurso público y político-, Rampton muestra cómo la lengua desempeña un rol significativo en las políticas culturales cotidianas. 
nómeno requiere de constante movimiento entre límites étnicos y sociales tradicionalmente establecidos, así como el desdibujamiento de éstos (1995). Los constantes procesos diaspóricos y los avances tecnológicos en un mundo altamente interconectado (Cf. Appadurai, 1996) hacen que la idea de una lingüística fundamentada en límites geopolíticos resulte de cada vez menos viable para propósitos de análisis social y cultural. El interés se centra ahora en la fragmentación, la multiplicidad y la contradicción: "in the boundaries of inclusion and exclusion, and in the flows of people, knowledge, texts, images, and objects across social and geographical space" (Rampton, 1995: 2) alejándose de una deseada totalidad o un concepto imaginado de estado-nación. Con este fin, Jan Blommaert propone una sociolingüística de la movilidad que aborda la lengua como un fenómeno en constante movimiento y que se enmarca en la perspectiva de redes trans-contextuales, flujos y corrientes, estratos verticales, y múltiples espacios (más allá del estudio cambiante de patrones lingüísticos fijados en una localidad o entre dos regiones) y tiempos (más allá de estudios de transmisión generacional) (Blommaert, 2010; Cf. Heller, 2007); o sea, una sociolingüística más acorde a la actualidad que el enfoque sincrónico estructuralista del estudio de la lengua en el campo de la lingüística moderna.

El cruce lingüístico en la producción artística grancaribeña crea una estética de la multiplicidad que desnaturaliza la noción de las identidades con guion, representada lingüísticamente en el concepto de code-switching, en el cual persiste la presuposición de que los hablantes bilingües se mueven entre diferentes identidades lingüísticamente marcadas, las cuales se asocian a imaginarios nacionales establecidos -normalmente una lengua perteneciente a una comunidad minoritaria avis-à-vis la lengua institucionalizada- (Rampton, 2010: 6-7; Cf. 1995: 9). Blommaert agrega que los estudios convencionales de alternancia de código no hacen justicia 
a la complejidad de los repertorios multilingües, multi-escala, y súper diversos que ocurren en micro-ambientes locales concretos en un mundo globalizado (2010: 12, 42). En contraste, la noción de cruce(s) lingüístico(s) da cabida a prácticas lingüísticas transicionales, complejas y no estáticas capaces de representar identidades culturales con configuraciones múltiples y diversas escalas verticales en cuestiones de género, clase y etnicidad. En las narrativas grancaribeñas, el cruce lingüístico -marco teórico de mi análisisdesnaturaliza el español de la cultura caribeña de origen (junto con todos los preceptos normativos que acompañan a esta lengua en el Caribe) y el inglés de EEUU que se imagina homogéneo, a la vez que desfigura los límites de las identidades lingüístico-culturales y nacionales que se imaginan a cada lado del guion hacia una visión de estos conceptos como formaciones heterogéneas afines a la lógica posmoderna. ${ }^{8}$

Con todo lo anteriormente señalado, las autoras grancaribeñas se agruparían en una comunidad de práctica literaria unida por

${ }^{8}$ Como demuestran la mayoría de los estudios sociolingüísticos sobre el cambio de código (Cf. Koike, 1987; Toribio, 2004; Zentella, 1997), en los EEUU este fenómeno lingüístico responde principalmente a factores pragmático-discursivos (Cf. Klee y Lynch, 2009: 226) y, en menor medida, a la falta de competencia lingüística en cada una de las lenguas en juego. En el caso de las autoras grancaribeñas, el cruce lingüístico se da por motivos tanto formales como funcionales, pero también por razones estéticas e ideológicas. Algunas veces, el objetivo de las escritoras es simplemente recordar al lector que la historia ocurre en español (Lynch, 2011). En otras ocasiones, estas cruzan lengua(je)s con la intención de crear un efecto estético en particular (multiplicidad, opacidad). La mayoría de las veces, combinan forma y funcionalidad hacia un fin ideológico: reclamar el derecho a la opacidad (Glissant, 1997) o crear "a marker of Hispanic identity" (Pérez Rosario, 2010: 11). Aunque algunos de estos motivos coinciden con los que nos muestran los estudios de casos de code-switching, prefiero evitar el término 'código' en este artículo puesto que, como afirma Monica Heller, el propio concepto de 'código' implica sistemas lingüísticos delimitados y autónomos (2007: 7) junto con sus imaginarios nacionales correspondientes. 
un virtuosismo lingüístico de estrategias compartidas, el cual se convierte en un tipo de branding-léxico en la mayoría de los casos y siempre ideológico- constituido por el ya mencionado fenómeno del cruce lingüístico en sus obras. La comunidad de práctica literaria unida por un branding lingüístico que se presenta en este artículo no se basa ni en una lengua única y unificada ni en una subjetividad o territorio nacional común, sino que el cruce lingüístico como práctica posmoderna; agrupa a las autoras en una comunidad de práctica translocal e incluso posnacional que propone una transformación social, y que no se forma con base en aspectos cronológicos o espaciales, sino lingüísticos e ideológicamente feministas, dándole un compartido sentido político a la estética de multiplicidad que crean. ${ }^{9}$

Mark Sebba (2015) acuña el término branding para referirse a un elemento gráfico/visual concreto de la lengua escrita que se

${ }^{9} \mathrm{Mi}$ marco teórico-conceptual en lo que se refiere a la comunidad de práctica literaria que forman las escritoras de estudio va de la mano con la conceptualización que Rampton hace del término de Lave y Wenger. Rampton concibe comunidades de práctica que se interesan en cómo "notionally multiple memberships and identities get constructed and integrated in social practice" en vez de "treating people just aggregations of 'tickbox' social variables as independent modules" (2010: 5) como lleva haciendo la tradicional lingüística de distribución. Si reconocemos que el uso de la lengua se halla intrínsecamente ligado a la organización social, entonces, la transformación de la lengua en los textos grancaribeños de estudio abre una puerta a un espacio donde modos de expresión no dicotómicos, categorías sociales alternativas y otros tipos de agrupaciones literarias tienen cabida. Se trata de un uso heterogéneo de la lengua dentro de un grupo complejo de textos y autoras que a menudo se solidarizan y, otras tantas veces, se contradicen. Cabe destacar que, metodológicamente, combino la perspectiva de la comunidad de práctica con el enfoque de la ideología de la lengua en sociolingüística, cuyo ángulo de visión me permite analizar el concepto de 'comunidad' como constructo político, que en este caso cuestiona supuestos ideológicos que la conceptualización del guion implica y transmite, además de que señala las exclusiones que este concepto acarrea. 
convierte en un emblema para el grupo de individuos que lo usan en sus prácticas lingüísticas escritas. Incluso aquellas personas que no conocen el idioma en cuestión, pueden identificar dicho elemento. ${ }^{10}$ En Dreaming in Cuban y Memory Mambo, el español se inserta en el discurso en inglés con el uso de cursivas - "as if to emphasize their strangeness"- (Lauret, 2014: 2). La inclusión de léxico en español en textos escritos principalmente en inglés (o viceversa en el caso de la narrativa de Mayra Santos Febres o Aurora Arias, por ejemplo) con el uso de la marca visual de la cursiva puede entenderse como un tipo de branding-léxico- a través del cual estas escritoras crean una imagen para su comunidad de prácticas discursivas. En su segunda novela, Days of Awe (2001), Obejas elimina la cursiva con el propósito de naturalizar el cruce lingüístico. La autora confiesa que la cursiva enfatizaba "the other rather than the commonality" y que hoy en día palabras como 'bodega' o 'intifada' deberían considerarse parte del vocabulario americano (Obejas, 2001). La evitación de algún tipo de letra de imprenta para marcar el cruce lingüístico se convierte entonces en un proceso sub-branding dentro del branding principal, lo que termina como un mayor intento de revelar una representatividad Latina heterogénea en los EEUU. Según sugiere Sebba, el hecho de que exista la opción de elegir entre dos marcas (brands)-usar las cursivas o no- con la misma funcionalidad ideológica final incrementa el potencial de marcado de identidad de la marca inicial. El imaginado monolingüe y monocultural lector anglófono de estos textos (i.e. un lector ideal-izado siguiendo los preceptos nacionales modernos) puede no entender el significado de las inserciones

${ }^{10}$ Como ejemplo de branding, ortográfico en este caso, Sebba menciona el debate que se creara alrededor del grafema ' $k$ ' en el criollo haitiano, el tagalog en las Filipinas y el vasco en España. En concreto, la ' $k$ ' se usa de forma subversiva como reemplazo de la 'c' castellana como marca lingüística de la contracultura activista vasca para hacer que el español se parezca al vasco. 
léxicas en español, pero con seguridad las reconoce como tales y las interpreta como una práctica ideológico-cultural compartida.

En las novelas de García y Obejas, el branding no resulta completamente ininteligible para el público anglo monolingüe (es decir, la comprensión de las novelas no requiere lectores bilingües), aunque sí sugiere un sentido de opacidad para ciertos discursos estrictos, elitistas y puristas de 'americanidad' y 'cubanidad', como apunta Frances R. Aparicio con el concepto de 'tropicalización' (1994: 796). ${ }^{11}$ Aparicio confirma que esta literatura privilegia al lector ideal bilingüe y bicultural mientras que simultáneamente logra un equilibrio al negociar entre el público anglófono monolingüe y el latino (bilingüe). Agrega que una lectura monolingüe de los textos en cuestión sólo puede ser parcial y limitada (1994: 800) debido a la intencionada opacidad de estas novelas. A través de dicha opacidad, el lector monolingüe estadounidense, que ha sido históricamente el prototipo de la alfabetización cultural (1994: 800), queda relegado a un segundo plano o, alternativamente, se ve forzado a cruzar lenguas y culturas, además de aceptar la realidad heterogénea de los EEUU. ${ }^{12}$

En la novela de García, hallamos una explicación contextual de estos vocablos, sin embargo, otras veces no hay forma de saber el significado de la palabra por el contexto. En estos casos, el

${ }^{11} \mathrm{Al}$ tomar prestado uno de los títulos de Hernández Cruz, Aparicio define el proceso de 'tropicalización' en la narrativa 'US Latina' como "a cultural and discursive counter-movement developed by Latino/a communities that dialogizes homogeneous Anglo constructs of the Latino/as as Other" (1994: 796).

${ }^{12}$ Lourdes Torres corrobora que el lector destinatario de estas narrativas se imagina monolingüe en inglés, aunque es cierto que de cierta forma se favorece al lector bilingüe (1997: 77) y, en consecuencia, se arroja una lanza a favor de la multiplicidad lingüístico-cultural. Cabe recordar que, en su mayor parte, el hablante de herencia de español que podría interesarse por leer estas novelas ha recibido su educación formal (sino toda, casi en su totalidad) en inglés (Cf. Beaudrie y Fairclough, 2012). 
lector no recibe ninguna traducción o aclaración de la expresión en español. En Memory Mambo, el lector encuentra la definición y/o traducción, literal y libre, de las locuciones en español en un glosario en la parte final del libro (1996: 239). Obejas expone que

... [t] he glossary's about culture and history. It was a way to create context, for those readers who want it, without interrupting the flow of the story ... there are no footnotes, so the reader has to decide whether she wants to see if there's anything back there, in the glossary, about whatever it is she thinks she may have missed, or wants to know more about... The italics I'll never use again; the glossary, yes (Preziuso, 2010: 1).

De esta forma, estas narrativas son, en palabras de Torres, "easily accessed, transparent and cushioned" (1997: 79). Además, considerando lo que ya he argumentado con anterioridad, me adhiero a la idea de que "by signaling otherness through their language choice [these grancaribeña writers are not] consciously trying to exclude some readers" (Torres, 1997: 90). Más bien todo lo contrario, están forzando a estos lectores a cruzar lengua(je)s, al tiempo que animan a los más curiosos a ver "if there is anything back there". Cristina García sugiere esta intencionalidad al señalar que las inserciones en español en sus obras funcionan "like a punch" para recordarle a los lectores que, aunque estén escritas en inglés, sus historias ocurren en español (Lynch, 2011). Este "punch" va dirigido tanto al lector estadounidense sin guion para recordarle la presencia latina en los EEUU, como al lector cubano-americano que impone su mito -"created in exile, a group hallucination" (Obejas, 1996: 25)- de lo que debería ser y significar la cubanidad. Maria Lauret confirma que estas palabras se integran en la narrativa con el objetivo de "disrupt, enchant, occlude or highlight the taken-for-granted English of American literature and can thereby perform wonders of poetic signification as well as cultural 
critique” (2014: 2). Además, la mayoría de estos vocablos o expresiones en espańol en estos textos emergen en los diálogos de los personajes con un propósito mimético o emocional (Cf. Pavlenko, 2006, 2007). García apunta que, asimismo, son términos que en inglés perderían ciertas connotaciones, referentes culturales y/o cargas semánticas (Cf. Torres, 1997: 77; Otheguy, 2003: 16). En Memory Mambo hallamos, por ejemplo, las siguientes inserciones en español: léxico de la familia como 'tío' y 'tía' o 'primo' y 'prima'; expresiones coloquiales como 'qué carajo'; vocablos con una carga semántica político-cultural importante, como 'independentista' (término perteneciente al contexto socio-político puertorriqueño); palabras relacionadas con la gastronomía, 'cafesito' o 'ropa vieja', e insultos -que implican una destacada carga emocional, además de cultural- como 'comemierda'. En Dreaming in Cuban, hallamos tipos de vivienda específicos de Cuba, como 'finca'; 'novelas', con un claro componente cultural; motes cariñosos como 'mi cielo'; términos familiares como 'mi hija'; entre muchas otras palabras y expresiones. Es un léxico dentro del campo semántico de la cotidianeidad, de ahí que el branding lingüístico podría ser fácilmente reconocido por el lector anglófono con nociones extremadamente básicas del español.

Así, no sólo las escritoras, sino también los críticos literarios y demás lectores, identifican las obras grancaribeñas como pertenecientes a un grupo concreto por medio de la atribución de la práctica del cruce lingüístico como característica propia de la narrativa de esta comunidad literaria. La atribución de esta peculiaridad lingüística a la producción artística de este grupo de escritoras implica la no-atribución de la misma particularidad a las producciones creativas de otros grupos de escritores que poseen una percepción más estructuralista de la lengua (creativa) y un marco ideológico diferente en lo que se refiere a los conceptos de nación(alidad) e identidad. Estos otros grupos -o sus lectores 
y críticos literarios- tienden a reaccionar negativamente ante la marca de las grancaribeñas e incluso podrían intentar corregirla (Cf. Sebba). De cualquier forma, el cruce lingüístico se convierte en un signo icónico de estas obras y sus escritoras, pues sus prácticas de escritura representan la naturaleza fluida de éstas y su común ideología de multiplicidad. ${ }^{13}$ Cabe destacar que a través de la condición discursiva y visual del branding no sólo consiguen las escritoras grancaribeñas llevar a cabo un proceso de desarticulación identitaria, sino que además consiguen un "distinctiveness or unique selling point” (Sebba, 2015: 213). Comience la agencia de este proceso ideológico con las escritoras o con sus lectores y sea el branding lingüístico bienvenido o rechazado por el público lector, los cruces lingüísticos que crean las autoras se convierten estratégicamente en una marca ideológica y de identidad que les otorga tanto a ellas como a su literatura visibilidad en el mercado literario local y global.

Lauret sugiere que el multilingüismo intra-textual en este tipo de textos podría acabar siendo característico de la literatura global (2014: 5), pero además revela la heterogeneidad local y trae al centro discursivo prácticas sociales periféricas, desafiando así las corrientes globales (mientras que las re-visita y participa en ellas). Asimismo, esta estrategia lingüística cuestiona discursos hegemónicos concomitantes de poder y códigos literarios fundacionales establecidos por escritores canónicos siguiendo preceptos patriarcales. Así, en lo que se refiere a cuestiones de cánones literarios, me opongo a la idea de que "by choosing either Spanish or English

${ }^{13}$ Considerando que la lengua escrita implica necesariamente la creación de una imagen, Sebba rescata la noción de iconization de Irvine y Gal como proceso ideológico por el cual las características lingüísticas que identifican a los grupos sociales se convierten en representaciones icónicas de éstos, como si dichas particularidades representaran o mostraran la naturaleza inherente del grupo en cuestión (37). 
these writers intentionally give up the idea of belonging to intellectual communities that are essential for their creative survival" (Álvarez-Borland, 1998: 9; el énfasis es mío). A través del cruce lingüístico, estas escritoras se insertan en, y gradualmente transforman, tradiciones literarias en Cuba y los EEUU. Tradiciones que se unen en la creación de una literatura multilingüe. Por ejemplo, en su introducción a la antología cubana de la cual es editora, Estatuas de sal. Cuentistas cubanas contemporáneas, Mirta Yáñez declara que "la otra orilla' de la cuentística cubana" también pertenece a la literatura de la isla -"quiéranlo o no"-, aunque se escriba en una "lengua intermedia, o llanamente en el idioma del país donde se han formado, en inglés” (39). Para Yáñez, que su narrativa esté escrita en inglés no excluye a autoras 'en el exilio' como Obejas y García de la academia cubana. La acotación "quiéranlo o no" muestra la polémica existente a la hora de unir o distinguir entre las escritoras de cada orilla en un momento en el que "la dilucidación de este complejo fenómeno sólo ha comenzado por ambas partes" (39). Sin embargo, cabe resaltar, que el cuento de Obejas "We Came All the Way from Cuba So You Could Dress Like This?” (1994) se publica en esta compilación de narrativa breve de Yáñez, co-editada con Marilyn Bobes, como “¿Vinimos desde Cuba para que te pudieras vestir así?”

Tal como apunta Karen S. Christian, ¿por qué deben ser los cañones literarios de Cuba y los EEUU mutuamente excluyentes? (2010: 20) ¿Por qué el hecho de escribir en un idioma u otro etiqueta inevitablemente estos trabajos en categorías literarias y nacionales concretas? (2010: 18) ¿Debería García, que casi no escribe en español, incluirse en un canon literario nacional diferente al que perteneciera Obejas, quien escribe más a menudo en ambas lenguas? Resulta harto complicado y problemático establecer una conexión directa entre lengua de creación e identidad (nacional) o 
cánones literarios en estas escritoras, como hace Álvarez-Borland al referirse a los escritores cubano-americanos que escriben en inglés:

Spanish means a link to [their] roots and to the traditions of the culture of [their] nation .... Those who have chosen to write in their native tongue feel that their literary production very much belongs to the tradition of Cuban letters and therefore want to be a part of this canon. In other words, their identity as writers neither comes from nor belongs to North American letters (1998: 153-154).

Como Christian indica, al hacer esta conexión entre lengua e identidad, Álvarez-Borland obvia la complejidad lingüística que presentan estos escritores (1998: 21). Su dependencia en categorías literarias estáticas y claramente demarcadas se aleja del quid de los textos que aquí analizo. Su noción de elección lingüística, junto con la concepción de la lengua española como representación de la lengua y nación 'original' de las autoras es problemática si tenemos en cuenta la índole translocal y posnacional de estas novelas.

En definitiva, el cruce lingüístico en la literatura de la Gran Cuba no debe interpretarse como un rechazo a las letras e identidades culturales cubanas y/o estadounidenses, sino como una redefinición de lo que estas debieran significar. Nos hallamos ante una reconceptualización del imaginario nacional monolingüe y monolítico en ambas tradiciones literarias. La funcionalidad y los efectos semánticos y estéticos del cruce lingüístico, junto con los aspectos temáticos de sus novelas -que analizo en profundidad en el caso de Dreaming in Cuban en la sección siguiente-resaltan la idiosincrasia posnacional de esta producción artística. 


\section{Dreaming in Cuban de Cristina García: "Painting Is its Own Language"}

Si desde la era moderna la lengua ha ido inextricablemente ligada a proyectos de nación(alidad), y si se tiene en cuenta que estos se construyen y mantienen sobre una base patriarcal, entonces el cruce lingüístico en la comunidad de práctica a la que pertenecen estas escritoras de la Gran Cuba ofrece una alternativa feminista sumamente apropiada al concepto espacial y político del Estadonación y a los roles de masculinidad y feminidad que se le asocian. George Yúdice argumenta que el feminismo como discurso "es deconstructor de todo nacionalismo, [porque] va más allá del nacionalismo" (1986: 1) y Jean Franco asevera que las mujeres siempre han tenido un rol secundario en la construcción de naciones puesto que "toda identidad nacional es, en esencia, una identidad masculina" (1997: 81). A este respecto, además de re-visitar la ideología de 'una nación, una cultura, una lengua', los textos gran caribeños reinterpretan lo que pueden considerarse macronarrativas androcéntricas. Por ello, la trama de la opera prima de Cristina García presta especial atención a la genealogía femenina de sus personajes porque "[s]o much of history is written by and about men” (Shibuya Brown, 1993: 250).

Abuela Celia encabeza la genealogía. Su fallecido esposo, Jorge del Pino, tan sólo aparece en las cartas que ésta escribe a un amante previo, Gustavo, y como figura fantasmal en los sueños de su hija Lourdes. A pesar de sentirse atrapada en Cuba como una prisionera, Celia, ferviente simpatizante del régimen castrista, nunca sale de la isla (49). Esto a pesar de que se queda sola cuando su hija Lourdes emigra a Nueva York con su esposo Rufino y su hija Pilar en oposición al régimen, al igual que su marido, quien se muda a EEUU en busca de tratamiento médico. Pilar, el alter ego de García según ha admitido la propia autora (Shibuya Brown, 1993: 
251), es hija de la revolución cubana. Criada en Nueva York bajo los mandatos anti-comunistas y asimilacionistas de su madre, Pilar sueña con una Cuba que apenas recuerda.

La joven se halla en plena crisis de identidad provocada por un sentimiento de desplazamiento y por una incesante lucha para que la sociedad no la constrińa a una forzada bipolaridad (inglés/español, cubana/estadounidense). Por medio de un viaje al supuesto 'origen', la protagonista se halla en el proceso de reconocimiento de su identidad múltiple, compleja y contradictoria en contraposición a aquellos discursos ideológicos que la obligan a ser una mujer americana o cubana que cumple determinados preceptos; o que esperan que se sienta anclada en un guion entre imaginarios nacionales y culturales, además de roles de género con los que no se identifica. Este viaje al 'origen' únicamente confirma que la respuesta a su búsqueda identitaria no se encuentra aquí o allí, sino aquí, allí y en múltiples lados. Justamente, García muestra experiencias femeninas multifacéticas y particulares en relación al exilio y al sentimiento de desplazamiento. Aunque inicialmente se presente como un conflicto, para Pilar la fragmentación lingüística e identitaria no acabará siendo una experiencia y carga traumática como lo fuera para la generación de sus padres, cuyas narrativas imperantes va a desafiar.

En su afán por expresar su visión de mundo en un lenguaje que no sea ni el inglés ni el español, que la ciñen a imaginarios homogéneos de identidad, Pilar busca otro modo de expresión heterogéneo, y lo encuentra en la pintura: "Painting is its own language... Translations just confuse it, dilute it, like words going from Spanish to English... Who needs words when colors and lines conjure up their own language?" (1993: 59, 139). Como explica Álvarez-Borland, "[t]o the dilemma of language loss, Pilar finds that visual images communicate meaning much more effectively than does language" (1994: 46). De esta forma, en el tropo del arte 
pictórico, se materializa el proyecto que lleva a cabo a nivel formal la autora con sus prácticas lingüísticas. A través de la pintura, Pilar halla la representación visual ideal del cruce lingüístico como la expresión artística que funciona como una micro-narrativa que consigue reemplazar macro-retóricas patriarcales que llevan narrando la(s) historia(s) de las mujeres en el Gran Caribe en una suerte de globalización vernácula; i.e.m una muestra de la interacción entre lo local y lo global que explica cómo las macro-narrativas modernas "[are] often punctuated, interrogated, and domesticated by the micronarratives of film, television, music, and other expressive forms" (Appadurai, 1996: 10).

Dreaming in Cuban se sitúa en Nueva York, una urbe multiétnica que ayuda a imaginar y crear una Cuba en dispersión que es heterogénea y que posee múltiples gradientes y subjetividades diaspóricas. Este enclave no se define por la idea del regreso (o no) al lugar de origen, la cual ocupa un lugar prominente en algunos (que no todos) discursos homogéneos dentro de la comunidad diaspórica cubana en Miami, por ejemplo. Tal como declara Martínez-San Miguel, "esta insistencia en el viaje o el retorno sigue privilegiando el vínculo con un territorio nacional único o la experiencia de los migrantes de primera generación como fenómenos que autentican identificaciones culturales" (2003: 37). Pilar no se identifica con estos discursos que perpetúa la primera generación de exiliados cubanos a los EEUU, lo cual se convierte en el motivo principal de roce entre la protagonista y su madre Lourdes. A Pilar le faltan aquellos referentes culturales que su madre repudia y piensa que encontrará esos fragmentos perdidos de su ser en Cuba y mediante la figura de su Abuela Celia, ya que ésta es la única conexión femenina que posee con la isla y con los silencios que llenan su historia familiar. La Abuela juega en la novela el papel de rellenar los huecos que la primera generación de exiliados en los EEUU ha dejado en Pilar. 
En el capítulo que abre Dreaming in Cuban, una voz narrativa en tercera persona describe a Celia solitaria mientras se lamenta del destierro en su propia tierra, por el cual la Revolución la ha separado de su familia. Con todo, se siente en deuda con la causa revolucionaria hasta el punto de ofrecer sus servicios al líder desde su casa frente al mar como punto de vigilancia principal en Santa Teresa del Mar, mientras se lamenta de que su nieta Pilar la escriba desde Brooklyn "in a Spanish that is no longer hers" (1993: 7).

Lengua e identidad se entremezclan en la novela: la tercera y última sección del libro se titula "The Languages Lost", haciendo mención al español de Pilar "that is no longers hers" y a aquellos aspectos de su vida en Cuba que la han perdido. Jacques Derrida comienza su Monolingualism of the Other afirmando que el novelista marroquí Abdelkebir Khatibi habla de su lengua materna en francés. Khatibi confiesa que su lengua materna lo ha perdido (35), en vez de ser él quien ha perdido su lengua, debido a lo que Derrida denomina 'la hegemonía de lo homogéneo', i.e. el monolingüismo impuesto por los poderes coloniales. Derrida añade, "certainly, he [Khatibi] evokes a language of origin which has perhaps 'lost' him, but which he himself has not lost ... He has a mother tongue plus another language" (36). De la misma forma, Pilar inicialmente cree que necesita recuperar la lengua que ha perdido para conseguir comprender los silencios que ha heredado y que fragmentan su propia historia personal y social. Pero una vez que consigue dar todas las pinceladas que configuran su autorretrato (abstracto), se da cuenta de que es la lengua la que quizás la ha perdido a ella, no al revés. Asimismo, logra reconocer que la única forma de expresión con la que realmente sabe expresarse y que de verdad la representa es la pintura, pues es fluida y se halla libre de la carga que tanto el inglés como el español han llevado para ella hasta el momento. Curiosamente va a ser su abuela -i.e. su principio cubano-quien la animará a tomar clases de pintura 
(29), lo cual va a remediar en Pilar la conmoción de la primeramente asumida pérdida lingüística. Ya lo expone Álvarez Borland, en Pilar "García dramatizes the anxiety of the search for voice and identity. The expression of fracture together with the demand for freedom become explicit in the need to be freed from maternal ties, political stereotypes, geographic locations, and traditional language. In this sense, painting will be the only conduct for expression and liberation" (1998: 137).

$\mathrm{El}$ arte pictórico de Pilar no es realista. Sus retratos no son estáticos y bien definidos, sino que los completan "knots and whorls of red that resemble nothing” (7), según los describe Abuela Celia sin juzgar el talento artístico de su nieta. Lourdes, por el contrario, cree que el arte abstracto de Pilar, "violent-looking with clotted swirls of red" (29), es mórbido, sin pensar que este tipo de representación artística se ha convertido en la lengua de su hija. La propia Pilar lo explica así: "Abstract painting is more up my alley. I feel more comfortable with it, more directly connected to my emotions" (233). Este modo de expresión se convierte para Pilar en una vía de escape que se aleja de pensamientos binarios e imaginarios nacionales e identitarios impuestos. El entendimiento estético de Pilar se asemeja al concepto de 'paraestética' de Carroll (1987), que contrasta con los patrones estéticos clásicos. Felski explica,

while classical aesthetics speaks of the harmony, totality, and integrity of the art work, paraesthetics prefers the language of contradiction and undecidability. ... it crystallizes and comments self-consciously on a general cultural condition: the end of metaphysics, the lack of foundations, and the slippery and indeterminate nature of language and communication (181).

Los nudos y espirales que pinta Pilar encarnan la deconstrucción de una ontología basada en la linealidad y las oposiciones 
de dos elementos a favor de una conceptualización posmoderna del mundo, que se interpreta como una matriz de relaciones: un espacio entrecruzado del cual emergen y se entrelazan múltiples significaciones (red knots and swirls). Más que expresar una verdad universal de su identidad como mujer, el arte y la estética de la multiplicidad de Pilar cuestionan y subvierten cualquier supuesto universal de identidad de género.

La violencia en sus cuadros -el color rojo simbolizando la sangre- representa la violencia que se le ha infligido a las diversas protagonistas debido a sus pérdidas lingüísticas, su sentimiento de dislocación en contextos nacionales y cuestiones de género, además de los traumas estructurados e ideologías predeterminadas impuestas por las generaciones previas. De hecho, Lourdes es la figura que más silencia a Pilar, quien le corta las alas y desaprueba su modo de expresión. A modo de ejemplo, Lourdes se niega a permitirle a Pilar que acepte una beca para estudiar en una escuela de arte en Manhattan (29). Además, al castigar a su hija, lo hace prohibiéndole pintar durante un mes (20). Pareciera que Lourdes perpetúa una visión de mundo patriarcal, adoptando para ella misma un tipo de masculinidad hegemónica tradicional en su entorno. Su carácter dominante silencia incluso a su esposo Rufino, el padre de Pilar. Rufino, más débil de personalidad, se ve incapaz de darle a su esposa lo que ella necesita (21) y, desde que emigran, nunca llega a adaptarse a la ciudad de Nueva York (129-30). En cambio, Lourdes asimila desde el principio su nueva realidad, llevada por su rechazo a los 'privilegios' de haberse casado con un Puente. Se niega a pasarse el resto de sus días grabando cuberterías, viendo telenovelas y perfumándose las muñecas en Habana, Hialeah o La Pequeña Habana, Miami. "Lourdes knew she could never be this kind of woman," explica la voz narrativa (130). Reniega del tipo de comunidad de mujeres en el exilio que eternizan los modelos femeninos tradicionales de su Cuba natal. Más bien, en los EEUU, 
Lourdes y Rufino intercambian roles de género patriarcales, mientras que siguen sin abandonar los roles binarios. Lourdes lleva a cabo su propia revolución feminista para convertirse en una figura castrante para Rufino. Ella es la que lleva los pantalones en casa literal y metafóricamente hablando-. Mientras Rufino se queda en su taller de casa, Lourdes provee: regenta una panadería y tiene un segundo trabajo como auxiliar de policía. Lo que más disfruta de este último es llevar los zapatos negros de suela gruesa del uniforme: "These shoes are power. If women wore shoes like these, she thinks, they wouldn't worry so much about more abstract equalities" (127). Lourdes se reinventa en los márgenes de las normas de género establecidas para mujeres de su clase social en su contexto cubano. Cuando todavía vivía en Cuba, fue brutalmente violada por un soldado que la dejó embarazada y servía a la Revolución. Tiempo después abortó al caerse de su caballo. Ambos episodios traumáticos ocurrieron mientras Lourdes intentaba proteger su propiedad de las manos de los soldados del gobierno revolucionario. Con el fin de recuperarse y protegerse de estos traumas, Lourdes opta por transgredir roles de género y desempeñar el papel que la sociedad patriarcal asigna al hombre. Este tipo de travestismo en Lourdes, junto con su disrupción de roles genéricos convencionales, ofrece a Pilar un modelo de mujer que no se ajusta a los que la sociedad usualmente atribuye.

No obstante, éste no es el modelo que Pilar desea seguir. De hecho, Lourdes y Pilar tienen problemas de comunicación. Lourdes llora la muerte del hijo que nunca nació, pero aún así sueña con conversaciones con él de la misma manera en que Rufino habla con Pilar, por compañerismo (129). Es cierto que Rufino y Pilar se llevan muy bien y son capaces de comunicarse sin problemas; de hecho, gracias a su padre, quien logra convencer a su madre "in his unobtrusive way" (29), Pilar puede finalmente matricularse a la escuela de arte en Manhattan. Rufino incluso le construye un 
estudio en la casa donde puede pintar y expresarse libremente, de acuerdo con el estilo artístico que elige:

For some reason I think about Jacoba Van Heemskerck, a Dutch expressionist painter I've become interested in lately. Her paintings feel organic to me, like breathing abstractions of color. She refused to title her paintings (much less do patriotic murals for her mother's bakery) and numbered her works instead. I mean, who needs words when colors and lines conjure up their own language? That's what I want to do with my paintings, find a unique language, obliterate the clichés (139).

Jacoba Van Heemskerck fue alumna de su propio padre, también artista. De igual forma, Pilar comparte la pasión por la creatividad con Rufino, quien planea ideas brillantes de artefactos e inventos $(30,131,65)$. El taller de su padre y su estudio están uno al lado del otro. Sin embargo, la creatividad de ambos artistas se ve minada por la figura dominante de Lourdes, que hace sonar una campana cuando requiere que padre e hija dejen de hacer lo que estén haciendo en el taller y estudio, respectivamente.

Rufino, cabeza de familia en Cuba, es incapaz de reinventarse como inmigrante. Aunque intenta concebir y metafóricamente crear nuevas narrativas fundacionales en su pequeño taller (tal como se esperaría de su papel en un sistema patriarcal) no tiene éxito en ninguno de sus proyectos. Por ello, Lourdes asume el rol patriarcal, pues " $[\mathrm{u}]$ nlike her husband, she welcomes her adopted language, its possibilities for reinvention" (73). Mientras su marido permanece anclado en su imaginario cubano, Lourdes "wants no part of Cuba, ... which Lourdes claims never possessed her" (73). Cuba fue demasiado dolorosa para ella así que, aunque solía amar al país que la vio nacer y crecer, ahora prefiere olvidar a modo de supervivencia. Rufino en cambio, tal como su apellido indica (Puente), se establece entre Cuba y los EEUU, una condición in- 
termedia que Lourdes repudia; de ahí que ella elija cruzar al lado 'americano': "She [Lourdes] decides she has no patience for dreamers, for people who live between black and white" (129), expone la voz narrativa, y lo confirma Pilar: "[her] views are strictly blackand-white" (26). Observamos entonces cómo Lourdes representa los discursos autoritarios y polarizados -en términos socio-culturales e incluso raciales- de algunos exiliados cubanos de primera generación, especialmente en Miami (Cf. Alberts, 2005; Baez, 2001; de la Torre, 2003; Lynch, 2009; Portes y Stepick, 1993). ${ }^{14}$ Aunque, como indica la autora, Lourdes se establece en Nueva York, donde puede asimilarse más profundamente al frío estadounidense (69-70). Lourdes tiene claro su lado del guion. Ella está aquí, no allí; habla en inglés con acento, no en español; se siente americana, no cubana, y vive con orgullo una vida capitalista -en Nueva York, no Miami- no una vida comunista. Abuela Celia es la que permanece en el lado cubano del guion. En palabras de McAuliffe, Celia representa la "authoritative voice of Cuban culture" (2011: 4). Rufino, por su lado, ve el mundo desde el mismo guion, en el angustioso espacio intermedio entre cubano y americano, entre aquí y allí, entre español e inglés. Es Pilar la que concibe la posibilidad de escapar de esta epistemología bipolar, como ya hemos visto que hace la propia Cristina García con el uso de la lengua creativa: "Personally, I'm more interested in the ... shades of gray between these two extremes. ... There are many ways to be Cuban and I resist the notion that to be Cuban is to hold particular political views or act in certain circumscribed ways" (Shibuya Brown, 1993: 250). Mientras que Lourdes aborrece la ambigüedad (65), la ambigüedad es la lengua de Pilar, la cual consigue expresar a través

${ }^{14}$ Lourdes demuestra tener prejuicios raciales contra los irlandeses, italianos, judíos, negros y puertorriqueños; problematizando así la noción de una posible solidaridad pan-caribeña o pan-Latina y, a la vez, desdeńando las aspiraciones de igualdad de su hija Pilar. 
de sus cuadros. Pilar es cubana y americana, español e inglés, aquí y allí, un ser múltiple y complejo.

Debido a esta multiplicidad que inevitablemente requiere la transformación de la visión moderna del arte, Pilar accede a crear el mural que Lourdes le encarga para la inauguración de su segunda panadería, Yankee Doodle Bakery. Desde una perspectiva posmoderna, la protagonista desarticula narrativas nacionalistas y patrióticas por medio de su obra de arte. Es más, su arte le permite romper con los imaginarios nacionales y discursos imperantes con los que ha crecido: el imaginario nacional de la América conservadora de algunos miembros de la primera generación cubanoamericana en el exilio, como es el caso de su madre, y el imaginario estadounidense que se piensa 'puro', justo y libre. Su crítica a la hipocresía de la declaración de libertad estadounidense se hace patente en la representación pictórica de la Estatua de la Libertad en su mural. La versión de la Estatua de Pilar es según Luis, “a political commentary of how immigrants are treated in the United States ... [and there is] an allusion to the influence of the women's movement upon the artist" (1997: 218). Tal como era de esperar, Lourdes y sus invitados se sienten ofendidos al contemplar la obra de arte. ${ }^{15}$ Este episodio complica aún más la relación de Pilar con su madre, hasta que un día Pilar también sufre de abuso sexual en los EEUU, en Morningside Park en Nueva York. Sin compartirlo con su madre, y sin saber de su experiencia traumática similar, este incidente precipita la decisión de Pilar: ha llegado el momento en el que madre e hija vuelvan a Cuba.

Su vuelta al 'origen' la hace recuperar la relación con la lengua que (la) había perdido. Comienza a soñar en español (235) pero, al mismo tiempo, se da cuenta de que pertenece a Nueva York, a los EEUU: "I know now it's where I belong —not instead of here,

${ }^{15}$ Para una descripción e interpretación detallada y acertada del mural de la Estatua de la Libertad que crea Pilar vid. Luis (1997: 218). 
but more than here" (236). Para Lourdes, por el contrario, el viaje de vuelta a Cuba la reconcilia con su cubanidad. Incluso danza la conga llevada por el deseo de "show her daughter the artistry of true dancing" (224). En cambio, Pilar "moved jerkily, off the beat, sloppy and distracted. She dances like an American" (224). En este sentido, la joven se da cuenta de que su identidad no pertenece únicamente a una Cuba imaginada. Aunque en un principio idealizaba la isla y a su Abuela Celia, una vez que re-visita el país, Pilar también (re)conoce las contradicciones inherentes en el gobierno cubano. De hecho, su visita coincide con el éxodo de Mariel en 1980. Su primo Ivanito quiere salir del país y Pilar, escondiéndole algo por primera vez, no le cuenta a su Abuela que le ha visto en la embajada peruana listo para embarcar.

Es sólo a través de su arte que, una vez más, Pilar consigue hacer las paces con sus propios sentimientos. En el último capítulo, Abuela Celia le pide a Pilar que la pinte "in a flared red skirt like the flamenco dancers wear" (232) con algunos claveles. Cuando Pilar está a punto de comenzar el retrato, Abuela Celia le pregunta si esta vez va a quedarse. Mientras Pilar pinta, la Abuela le cuenta cómo antes de la Revolución "Cuba was a pathetic place, a parody of a country ... Freedom, Abuela tells [her], is nothing more than the right to a decent life" (233). No obstante, cuando Pilar le pregunta si podría pintar lo que ella quisiera en Cuba y Abuela Celia le contesta que sí siempre que no implique un ataque al régimen, Pilar duda de su discurso de libertad, al igual que duda del de los EEUU. Ante la famosa cita del líder en boca de su abuela: "Con la Revolución, todo; contra la Revolución, nada," Pilar se pregunta qué pensaría el líder de su arte. Imaginando la respuesta de Castro, la joven piensa que le rebatiría que el arte es la última revolución (235). Esta des-idealización del discurso cubano coincide con lo incómoda que se siente con el retrato clásico que está realizando para su abuela; pero es lo que la mujer desea y, al contrario de lo 
que hiciera con Lourdes, ha aprendido a respetar sus maneras y perspectivas aunque sean distintas de las suyas.

El final de la novela evidencia el vacío generacional e ideológico existente entre Lourdes, Pilar y Abuela Celia en términos de identidad nacional e ideología política en una familia separada por la Revolución de 1959 (Cf. Rodríguez-Mourelo, 2006: 162). No obstante, las une el lugar donde, como mujeres, las coloca cualquiera de los imaginarios a los que pertenecen. Cristina García ofrece la misma importancia literaria a los tres personajes, sin privilegiar a uno u otro $y$, aunque se identifica en mayor medida con Pilar, respeta y entiende los motivos que se esconden tras las decisiones vitales de Celia y de Lourdes. García expresa esta idea de la siguiente forma:

I grew up in a very black-and-white situation: My parents were virulently anti-Communist, and yet my relatives in Cuba were tremendous supporters of Communism, including members of my family who belong to the Communist Party. The trip in 1984 and the book, to some extent, were an act of reconciliation for the choices everybody made (Luis, 1997: 216).

Si las escritoras grancaribeñas desarticulaban estos discursos extremos y polarizados que las rodean a través de su escritura creativa, el modo expresión artístico de Pilar es asimismo su vía de articulación de la escala de grises que conforman su yo-mujer, además del medio con el que realiza una declaración feminista como las autoras de las novelas con su estética literaria. Pilar expresa sus preocupaciones a este respecto:

I think about all the women artists throughout history who managed to paint despite the odds against them. People still ask where all the important women painters are instead of looking at what they did paint and trying to understand their circumstances. Even supposedly knowledgeable and sensitive people react to good art 
by a woman as if it were an anomaly, a product of a freak nature or a direct result of her association with a male painter or mentor. Nobody's even heard of feminism in art school. The male teachers and students still call the shots and get the serious attention and the fellowships that further their careers. As for the women, we're supposed to make extra money modeling nude. What kind of bullshit revolution is that? (139-40).

En conformidad a estas ideas, cada vez que se le encarga a Pilar pintar un retrato clásico por aquellos que representan los imaginarios nacionales y de género que la han rodeado desde pequeña (cubano -Abuela Celia- retrato; americano -Lourdes- la Estatua de la Libertad), ella muestra su malestar. Preferiría hacerlo a su manera abstracta, que expresa mejor su identidad múltiple, ambigua y fluida. Así, al final de su proceso de maduración, Pilar aprende a cortar los lazos que la unían de forma idealizada a Abuela Celia. Ha dejado atrás el pensamiento binario entre un aquí y un allí (homeland) idealizado. Ha recuperado la lengua que creía perdida, la cual la ha recuperado a ella sin por ello hacerla decidir entre sus múltiples lengua(je)s.

Cuando en el pasaje final Celia se sumerge en el mar en lo que la mayoría han interpretado como un suicidio, la Abuela está recitando las mismas estrofas del poema "Paisaje" de Federico García Lorca que siempre recitó durante su turno de vigilancia sobre el mar, sólo que esta vez los versos aparecen traducidos al inglés (243). La única palabra escrita en español en el pasaje es "duende", que ahora no se encuentra en cursivas. Celia se da cuenta de que "she has never been farther than a hundred yards off the coast of Cuba" y empieza a considerar "her dream of sailing to Spain, to Granada, of striding through the night with nothing but a tambourine and too many carnations" (243). En el flamenco, tal como lo define Lorca en su famosa conferencia "La teoría y juego del duende" (1933), el "duende" es el alma irracional del arte; el lado 
oscuro de una obra de arte que va más allá de estilos convencionales. Interpreto esta parte final como el entendimiento por parte de Abuela Celia -el personaje que se halla más anclado en su propio imaginario nacional y su correspondiente geografía- de la futilidad de las ideologías binarias en cuestiones de nación(alidad), lengua y espacio. Incluso, ahora se siente preparada para cruzar al inglés y liberarse de la engañosa carga dicotómica español/inglés que sólo ha conseguido separarla de su familia. Al igual que Pilar, Celia sólo halla en el arte -en el duende de la poesía lorquiana- su modo de expresión, y sucumbe a él.

\section{Bibliografía}

Alberts, Heike, 2005, "Changes in Ethnic Solidarity in Cuban Miami”, The Geographical Review, núm. 95, pp. 231-248.

Álvarez-Borland, Isabel, 1998, Cuban-American Literature of Exile: From Person to Persona, University of Virginia Press, Virginia.

, 1994, "Displacements and Autobiography in Cuban-American Fiction" en World Literature Today, vol. 1, núm. 68, invierno, pp. 43-48.

Aparicio, Frances R., 1994, "On Sub-versive Signifiers: US Latina/o Writers Tropicalize English", American Literature, pp. 795-801.

Appadurai, Arjun, 1996, Modernity al Large, vol. 1, University of Minnesota Press, Minnesota.

Báez, Luis, 2001, Miami: donde el tiempo se detuvo. Revelaciones de Luis Ortega, Editorial de Ciencias Sociales, La Habana.

Beaudrie, Sara M., y Marta Fairclough (eds.), 2012, Spanish as a Heritage Language in the United States: The State of the Field, Georgetown University Press, Georgetown. 
Behar, Ruth, 1995, Bridges to Cuba. Puentes a Cuba, University of Michigan Press, Ann Arbor.

Blommaert, Jan, 2010, The Sociolinguistics of Globalization, Cambridge UP.

Bloomfield, Leonard, 1927, "Literate and Illiterate Speech", American Speech, t. 2, vol. 10, pp. 432-439.

Carroll, David, 1987, Paraesthetics: Foucault, Lyotard, Derrida, Methuen, Nueva York.

Christian, Karen S., 2010, "La lengua que se repite: Pushing the Boundaries of Cuban/American literature", Caribe: revista de cultura y literature, año 13, vol. 2, pp. 17-38.

Davies, Alan, 2003, The Native Speaker: Myth and Reality, Multilingual Matters Ltd, Estados Unidos.

De la Torre, Miguel, 2003, La Lucha for Cuba. Religion and Politics on the Streets of Miami, University of California Press, Berkeley.

Del Rio, Eduardo, 2008, One Island, Many Voices: Conversations with Cuban-American Writers, University of Arizona Press, Arizona.

Derrida, Jacques, 1998, Monolingualism of the Other, or, the Prosthesis of Origin, Stanford University Press, California.

Duany, Jorge, 2000, Reconstructing Cubanness: Changing Discourses of National Identity on the Island and in the Diaspora During the Twentieth Century, en Cuba, the Elusive Nation: Interpretations of National Identity, Damián J. Fernández y Madeline Cámara Betancourt (eds.), University Press of Florida, Tampa, pp. 17-42.

Felski, Rita, 2000, Doing Time: Feminist Theory and Postmodernist Culture, NYU Press, Nueva York.

Franco, Jean, 1997, "The nation as imagined community”, Cultural Politics, núm. 11, pp. 130-140. 
Fusco, Coco, 1995, English is Broken Here: Notes on Cultural Fusion in the Americas, New Press, Nueva York.

García, Cristina, 1993, Dreaming in Cuban, Ballantine Books, Nueva York.

García Lorca, Federico, 2004, "Juego y teoría del duende”, Litoral, núm. 238, pp. 150-157.

Glissant, Édouard, 1997, Poetics of Relation, The University of Michigan Press, Michigan.

Grosjean, François, 1982, Life with Two Languages: An Introduction to Bilingualism, Harvard University Press, Boston.

Heller, Monica, 2007, "Bilingualism as Ideology and Practice", en Bilingualism: A Social Approach, Heller, M. (ed.), Palgrave Macmillan, Basingstoke, pp. 1-22.

Hospital, Carolina, 1988, Cuban American Writers: Los Atrevidos, Ediciones Ellas/Linden Lane Press/ Co-Works, Princeton.

Irvine, Judith T., y Susan Gal, 2009, Language Ideology and Linguistic Differentiation, Linguistic Anthropology, Stanford University Press, Stanford.

Klee, Carol y Andrew Lynch, El español en contacto con otras lenguas, Georgetown University Press, Washington.

Koike, Dale April, 1987, "Code switching in the bilingual Chicano narrative", Hispania, pp. 148-154.

Lauret, Maria, 2014, Language Migration in American Literature, Bloomsbury Academic, Nueva York/Londres.

Luis, William, 1997, Dance Between Two Cultures: Latino Caribbean Literature Written in the United States, Vanderbilt University Press, Nashville/Londres.

Lynch, Andrew, 2009, "Expression of cultural standing in Miami: Cuban Spanish discourse about Fidel Castro and Cuba", Revista Internacional de Lingüistica Iberoamericana, pp. 21-48. 
, 2012, "Key Concepts for Theorizing Spanish as a Heritage", en Spanish As a Heritage Language in the United States: The State of the Field, pp. 79-100.

, 2011, "Novelist Cristina García Reflects on Language and Bilingualism (Andrew Lynch Interviews)". Disponible en: http://www.youtube.com/watch?v=kvi43MB_QuU

, 2003, "Toward a Theory of Heritage Language Acquisition: Spanish in the United States", pp. 25-50.

Manzor, Lillian, 2005, "Más allá del guion. El teatro usanocubano", en Teatro cubano actual, Ediciones Alarcos, La Habana, pp. VII-XXI.

Martínez-San Miguel, Yolanda, 2003, Caribe Two Ways: Cultura de la migración en el Caribe insular hispánico, Ediciones Callejón, San Juan.

McAuliffe, Samantha L., 2011, "Autoethnography and Garcia's Dreaming in Cuban", Comparative Literature and Culture, año 13, vol. 4. Disponible en: http://dx.doi.org/10.7771/14814374.1874 .

Obejas, Achy, 2009, Aguas y otros cuentos, Letras Cubanas, La Habana..

,2002, "Days of Awe by Achy Obejas. Interview with Achy Obejas", The Jewish Reader, julio. Disponible en: http:/www. yiddishbookcenter.org/node/327.

, 1996, Mambo Memory, Cleis Press, Pennsylvania/San Francisco.

Ortiz Cofer, Judith, 1990, Silent Dancing: A Partial Remembrance of a Puerto Rican Childhood, Arte Público Press, Houston.

Otheguy, Ricardo, 2003, "Las piedras nerudianas se tiran al norte: Meditaciones lingüísticas sobre Nueva York", Insula: Revista de letras y ciencias humanas, núm. 679, pp. 13-18. 
Pavlenko, Aneta, 2006, "Bilingual Selves", Bilingual Education and Bilingualism, núm. 56, pp. 1-33.

, 2007, Emotions and Multilingualism, Cambridge University Press, Cambridge.

Pérez Firmat, Gustavo, 2000, Cincuenta lecciones de exilio y desexilio, Ediciones Universal, Miami.

, 1995a, Life on the Hyphen: The Cuban-American Way, en Long Series in Latin American and Latino Art and Cuture, University of Texas Press, Texas.

, 1995b, Next Year in Cuba: A Cubano's Coming-of-Age in America, Anvhor Books, Nueva York.

Pérez Rosario, Vanessa, 2010, "Introduction: Historical Context of Caribbean Latino Literature", en Hispanic Caribbean Literature of Migration: Narratives of Displacement, Macmillan, Palgrave.

Piller, Ingrid, 2001, "Who, if Anyone, Is a Native Speaker", Anglistik: Mitteilungen des Verbandes Deutscher Anglisten, año 12, vol. 2, pp. 109-121.

Portes, Alejandro y Alex Stepick, 1993, City on the Edge: The Transformation of Miami, University of California, Berkeley.

Preziuso, Marika, 2010, "Interview with Achy Obejas, en Small Axe. Disponible en: http://smallaxe.net/wordpress3/interviews/2010/10/27/interview-with-achy-obejas/

Rampton, Ben, 1995, Crossing. Language and Ethnicity Among Adolescents, Longman, Nueva York/Londres

, 2013, "Language Crossing and the Redefinition of Reality", en Code-Switching in Conversation: Language, Interaction and Identity, Routledge, Londres, pp. 290-320.

, 2010, "Speech community", en Society and Language Use, pp. 274-303. 
Rengel, Patricia L., 2012, Essaying the Puerto Rican Nation: José Luis González, Luis Rafael Sánchez and Ana Lydia Vega, Tesis, University of Wisconsin, Madison.

Rodríguez-Mourelo, Belén, 2006, Encounters in Exile: Themes in the Narrative of the Cuban Diaspora, Aduana Vieja Press, España.

Santos Febres, Mayra, 1993, The Translocal Papers: Gender and Nation in Contemporary Puerto Rican Literature, Tesis, Cornell University, Nueva York.

Sebba, Mark, 2015, "Iconisation, Attribution and Branding in Orthography", Written Language \& Literacy, t. 18, vol. 2, pp. 208-227.

Shibuya Brown, Scott, 1993, "A Conversation with Cristina García”, en Dreaming in Cuban. Ballantine Books, Nueva York, pp. 249-256.

Toribio, Almeida Jacqueline, 2004, "Convergence as an Optimization Strategy in Bilingual Speech: Evidence from Code-Switching”, Bilingualism: Language and Cognition, t. 7, pp. 165-73.

Torres, Lourdes, 1997, “U. S. Latino/a Literature: Re-Creating America”, $A N Q$, t. 10, vol. 3, pp. 47-50.

Ween, Lori, 2003, “Translational Backformations: Authenticity and Language in Cuban America", Comparative Literature Studies, t. 40, vol. 2, pp. 127-141.

Wenger, Etienne, 1998, Communities of Practice: Learning, Meaning, and Identity, Cambridge University Press, Cambridge.

Yáñez, Mirta, y Marilyn Bobes, 2008, Estatuas de sal: Cuentistas cubanas contemporáneas: Panorama critico (1959-1995), Ediciones UNIÓN/Unión de Escritores y Artistas de Cuba, La Habana.

Yúdice, George, 1986, "El asalto a la marginalidad", Hispamérica, núm. 15 , diciembre, pp. 45-52. 
Valenciana, ISSN impresa: 2007-2538, ISSN electrónica: 2448-7295, núm. 22, julio-diciembre de 2018, pp. 217-257.

Zentella, Ana Celia, 1997, Growing up Bilingual: Puerto Rican Children in New York, Blackwell, Malden. 